\title{
ORIGINAL ARTICLE Colour ornamentation in the blue tit: quantitative genetic (co)variances across sexes
}

\author{
A Charmantier ${ }^{1}$, ME Wolak ${ }^{2}$, A Grégoire $^{1}$, A Fargevieille $^{1}$ and C Doutrelant ${ }^{1}$
}

Although secondary sexual traits are commonly more developed in males than females, in many animal species females also display elaborate ornaments or weaponry. Indirect selection on correlated traits in males and/or direct sexual or social selection in females are hypothesized to drive the evolution and maintenance of female ornaments. Yet, the relative roles of these evolutionary processes remain unidentified, because little is known about the genetic correlation that might exist between the ornaments of both sexes, and few estimates of sex-specific autosomal or sex-linked genetic variances are available. In this study, we used two wild blue tit populations with 9 years of measurements on two colour ornaments: one structurally based (blue crown) and one carotenoid based (yellow chest). We found significant autosomal heritability for the chromatic part of the structurally based colouration in both sexes, whereas carotenoid chroma was heritable only in males, and the achromatic part of both colour patches was mostly non heritable. Power limitations, which are probably common among most data sets collected so far in wild populations, prevented estimation of sex-linked genetic variance. Bivariate analyses revealed very strong cross-sex genetic correlations in all heritable traits, although the strength of these correlations was not related to the level of sexual dimorphism. In total, our results suggest that males and females share a majority of their genetic variation underlying colour ornamentation, and hence the evolution of these sex-specific traits may depend greatly on correlated responses to selection in the opposite sex.

Heredity (2017) 118, 125-134; doi:10.1038/hdy.2016.70; published online 31 August 2016

\section{INTRODUCTION}

Since Darwin's development of sexual selection theory (Darwin, 1871), theoretical and empirical work has greatly progressed towards explaining the mechanisms responsible for the evolution and maintenance of exaggerated male ornaments and weaponry (see, for example, Andersson, 1994; Savalli, 2001). Although secondary sexual characters are commonly more developed in males than females, in many animal species females also display elaborate ornaments (for example, conspicuous colours) or weaponry. After ignoring the issue for decades, evolutionary biologists have struggled to explain the evolution and maintenance of secondary sexual characteristics that are also exaggerated in females (Amundsen, 2000; Lebas, 2006; CluttonBrock, 2007; Kraaijeveld et al., 2007; Tobias et al., 2012). Two nonexclusive hypotheses have been suggested as explanations for the evolution and maintenance of female ornaments: (1) indirect selection for elaborate female traits through direct selection on correlated male traits (correlated response hypothesis) (Lande, 1980; Price, 1996) and (2) direct selection on female traits occurring through either femalefemale competition for mates (Dijkstra et al., 2008), male mate choice (Griggio et al., 2005; Byrne and Rice, 2006) or social competition for ecological resources (Heinsohn et al., 2005). Although each of these hypotheses has received some empirical support, there is currently no consensus about whether one plays a prevailing role, even within a given taxonomic group such as birds. A recent comparative analysis on 6000 species of passerines concluded that both female and male plumage colourations are more extravagant in larger species and in tropical species (Dale et al., 2015). Yet, the strength of sexual selection has antagonistic effects in the two sexes as it increases male colouration while decreasing female colouration (Dale et al., 2015), supporting the possibility of independent evolution as suggested by previous studies (Amundsen, 2000). The work by Dale et al. (2015) also confirms that the general focus on male ornamentation has limited our understanding of the evolution of colour ornaments in both sexes.

Even though the presence of strong cross-sex genetic covariances is a crucial assumption underlying the correlated response hypothesis, sex-specific estimates of key quantitative genetic (co)variances underlying secondary sexual traits that are expressed in both sexes have been conspicuously scarce in the empirical literature (but see Price and Burley, 1993; Price, 1996; Chenoweth and Blows, 2003; Roulin and Jensen, 2015). In particular, studies on the role of ornamentation have largely focussed on sexually dimorphic species while neglecting species with low or no sexual dimorphism (see reviews in Kraaijeveld et al., 2007; Poissant et al., 2010). In the absence of further investigation into the heritability of sexual ornaments/weapons and their cross-sex genetic covariance, no generality can be drawn from the present empirical data regarding the importance of the hypotheses cited above. For example, in the review of Poissant et al. (2010), only 14 out of 549 estimations of cross-sex genetic correlations concerned ornaments or weaponry and the strength of these correlations varied substantially

${ }^{1}$ Centre d'Ecologie Fonctionnelle et Evolutive, Université de Montpellier, UMR 5175 Campus CNRS, Montpellier, France and ${ }^{2}$ Institute of Biological and Environmental Sciences, School of Biological Sciences, Zoology Building, University of Aberdeen, Aberdeen, Scotland

Correspondence: Dr A Charmantier, Centre d'Ecologie Fonctionnelle et Evolutive, UMR 5175 Campus CNRS, 1919 Route de Mende, 34293 Montpellier Cedex 5, France.

E-mail: anne.charmantier@cefe.cnrs.fr

Received 17 March 2016; revised 8 July 2016; accepted 15 July 2016; published online 31 August 2016 
across taxa and across trait types (for example, between morphological traits and traits linked to communication).

In addition, theory for the evolution of sexual dimorphism also predicts that the degree of phenotypic difference between the sexes should be negatively associated with the cross-sex additive genetic covariance (and, to a certain extent, the cross-sex additive genetic correlation), and positively associated with the amount of sex-linked genetic variance (Fairbairn and Roff, 2006). Evidence supporting these predictions is presently very limited (Dean and Mank, 2014). Quantitative genetic analyses in natural populations based on longterm observations of individual phenotypes and relatedness (pedigrees) could offer a means to estimate sex-linked genetic variance. However, the large majority of studies in wild populations estimate additive genetic (co)variances while assuming only autosomal inheritance (Charmantier et al., 2014). Recent investigations on colour variation have revealed Z-linked genetic variance in the collared flycatcher Ficedulla albicollis (explaining $40 \%$ of total phenotypic variance in wing patch size; Husby et al., 2013), the barn owl Tyto alba (30\% of variance in eumelanic spot diameter; Larsen et al., 2014) and W-linked genetic variance in the zebra finch Taeniopygia guttata (2.6\% of variance in beak colouration; Evans et al., 2014). In many other cases however, investigations show no evidence for sex-linked genetic variance in colour ornamentation (for example, the Florida scrub-jay Aphelocoma coerulescens; Tringali et al., 2015). Overall, contributions of sex-linked genetic variance to phenotypic variance in sexually selected and morphological traits measured in pedigreed populations is usually weak, yet it is commonly acknowledged that this could be because of low power to distinguish autosomal from sexlinked genetic variance (Husby et al., 2013). It is indeed currently unclear whether wild population pedigrees used for quantitative genetic analyses confer sufficient power to disentangle autosomal additive genetic variance from other components of genetic variance (Wolak and Keller, 2014), as power analyses are not performed in these studies.

Colouration is often a sexually and/or socially selected trait that can signal individual quality and identity, as well as signal species identity, enhance crypsis, provide thermoregulatory benefits and protect against bacteria (Hill and McGraw, 2006), and is therefore central to an animal's fitness. However, to date, we know very little on the heritability of colouration (Svensson and Wong, 2011) or the genetic correlation that might exist between the sexes (Kraaijeveld et al., 2007; Roulin and Ducrest, 2013). Comparative analyses have shown that colouration can evolve conjointly or separately in the two sexes (Amundsen, 2000; Dale et al., 2015), but quantitative genetic studies of colouration are required to determine the main factors driving the observed sex-specific evolutionary patterns.

Although blue tits can appear sexually monomorphic to a human eye, spectrophotometry analyses have shown that blue tits from the subspecies Cyanistes caeruleus caeruleus are sexually dichromatic in the ultraviolet (UV) blue of the crown patch but monomorphic in their yellow carotenoid-based chest colouration (Andersson et al., 1998; Hunt et al., 1998; Doutrelant et al., 2008). Both male and female UV blue colouration influences intrasexual interactions (see, for example, Alonso-Alvarez et al., 2004; Rémy et al., 2010; but see Vedder et al., 2008), mutual mate choice (Hunt et al., 1999) and mate reproductive investments (see, for example, Sheldon et al., 1999; Limbourg et al., 2004; Kingma et al., 2009; but see Dreiss et al., 2006 for males and Limbourg et al., 2013 for females). In addition, male and female UV blue and yellow adult colouration is condition dependent (Doutrelant et al., 2012; but see Peters et al., 2011) and can be linked to parental investment or success (see, for example, Garcia-Navas et al., 2012;
Midamegbe et al., 2013) and to parasite levels (del Cerro et al., 2010). Overall, all these studies suggest that both UV blue and yellow colouration can be sexually selected in both sexes, yet Parker and colleagues (Parker et al., 2011; Parker, 2013) have recently challenged this view. Parker et al. (2011) found weak but contrasted evidence of fecundity selection on colouration for both sexes over 3 years. Following a meta-analysis that considered all previous studies with the same strength, regardless of the pertinence and robustness of their methodology, Parker (2013) further concluded that the sexual and/or social functions of blue and yellow colouration in blue tits remains to be demonstrated. This debate highlights the need for more studies on the colour patches in this species, and the examination of the cross-sex genetic correlation is an essential step to advance our understanding of the evolution in ornaments in both sexes. Despite many documented and proposed selective advantages to colour ornaments in blue tits, only three quantitative genetic studies have been conducted on colouration in this species (Johnsen et al., 2003; Hadfield et al., 2006a, 2007; Drobniak et al., 2013), showing low autosomal heritability for both types of colourations. Furthermore, the indirect selection hypothesis remains untested as there are no estimates to date of cross-sex additive genetic covariance.

We used 9 years of colour measures in long-term monitored blue tits located in a Mediterranean mainland population (subspecies C. $c$. caeruleus) and on the island of Corsica (subspecies C. c. ogliastrae) to investigate the sex-specific and cross-sex additive genetic (co)variances underlying colour ornamentation traits that show a gradient of sexual dimorphism, and have been suggested to be involved in intra- or inter-sexual selection. Colour features were measured in one structurally based (blue crown) and one carotenoid-based (yellow chest) ornament.

In the context of improving our understanding of the evolution and maintenance of sexual ornaments and the importance of genetic correlations in the evolution of female ornaments, our aims were originally threefold:

(1) Assessing whether there is autosomal and/or sex-linked genetic variation for colour ornamentation in the blue tit;

(2) Measuring the strength of cross-sex genetic covariances, with the particular aim to evaluate whether female ornament evolution could be driven by such covariances;

(3) Testing the theoretical predictions that the degree of sexual dimorphism is negatively associated with the cross-sex additive genetic covariance and positively associated with the amount of sex-linked genetic variance (Fairbairn and Roff, 2006; Poissant et al., 2010).

\section{MATERIALS AND METHODS}

\section{Sampling procedure and colour measurement}

Blue tits have been monitored in the Rouvière forest (mainland France) since 1991 and at two localities in Corsica since 1976 (Pirio) and 1994 (Muro). Details on these study sites can be found in (Blondel et al. (2006) and Charmantier et al. (2016). Blue tits from Corsica belong to a different subspecies from blue tits found in the French Mediterranean mainland. The distance between Muro and Pirio in Corsica is $25 \mathrm{~km}$. In order to improve our power for quantitative genetic models, individuals from these two valleys were pooled in one common Corsican data set. Supplementary Information A2 provides statistical justification for this choice based on a test for equality of additive genetic variances between the two populations.

Each year, breeding parents were captured in nest boxes between April and June. A small proportion of individuals were caught before the breeding period in January-March (in 2008-2009 and 2011-2013, $n=577$ or $15.9 \%$ of 
measures). Each bird was equipped with a uniquely numbered metal ring provided by the Museum National d'Histoire Naturelle in Paris, six blue feathers were collected from the bird's blue crown and eight yellow feathers from the yellow chest to allow colour measurements in the lab. Bird sex and age were determined based on the capture-recapture database or on the colour of wing coverts for unringed birds. Chicks were ringed after 9 days of age that allowed building social pedigrees for each population. Genotyping of parents and offspring in 2000-2003 has shown that up to 29.3\% (annual range: 18.2-29.3\%, Charmantier et al., 2004) of chicks were the result of extra-pair matings in Corsica, and 18.2\% (annual range: 11.5-18.2\%, Charmantier and Perret, 2004) on the mainland. The social pedigree used in this study was corrected for extra pair paternities only for chicks born in 2000-2003 in both populations. In these years, molecular genetic data allowed to identify $53 \%$ of extra-pair sires, whereas nonidentified genetic fathers were attributed a dummy identity. The Corsican pruned pedigree included 1507 individuals over 14 generations and the mainland pedigree 1233 individuals over 12 generations.

Feather colouration was measured in laboratory conditions, using a spectrometer (AVASPEC-2048, Avantes BV, Apeldoorn, Netherlands) and a deuterium-halogen light source (AVALIGHT-DH-S lamp, Avantes BV) covering the range 300-700 nm (Doutrelant et al., 2008, 2012) and kept at a constant angle of $90^{\circ}$ from the feathers. For each bird and colour patch (crown and chest), we computed the mean of six reflectance spectra taken on two sets of three blue and four yellow feathers (Doutrelant et al., 2008, 2012). We used the software Avicol v2 (Gomez, 2006) to compute chromatic and achromatic colour variables based on the shape of the spectra (Andersson et al., 1998; Doutrelant et al., 2008), following previous studies on blue tits in our populations (see, for example, Doutrelant et al., 2008, 2012) and others (see, for example, Alonso-Alvarez et al., 2004; but see Parker et al., 2011). For the UV blue crown colouration, we computed one achromatic variable: blue brightness (area under the reflectance curve divided by the width of the interval 300-700 nm); and two chromatic variables: blue hue (wavelength at maximal reflectance) and blue UV chroma (proportion of the total reflectance falling in the range 300-400 nm). Lower values of hue and higher values of UV chroma mean that the signal is stronger in the UV. For the yellow chest colouration, in addition to yellow brightness, we computed yellow chroma as
$\left(\mathrm{R}_{700}-\mathrm{R}_{450}\right) / \mathrm{R}_{700}$. Higher values of yellow chroma are linked to higher carotenoid contents in the plumage (Isaksson et al., 2008). We have shown previously that our measures of these five colour traits using a spectrometer are highly repeatable (see, for example, Doutrelant et al., 2008, 2012; Midamegbe et al., 2013), suggesting acceptable measurement error. Figure 1 displays average spectra for blue crown and yellow chest measures in 2011.

The complete data set included 3629 observations with at least one colour parameter measured ( $n=1659$ in Rouvière (mainland), $n=1035$ observations in Muro (Corsica) and $n=935$ in Pirio (Corsica)) for a total of 2177 birds (see Table 1 for detailed sampling efforts on males and females). Supplementary Figure S1 presents the distribution of each colour parameter in Rouvière and in Corsica and Supplementary Table S1 shows the phenotypic correlation between each pair of traits in the mainland and the Corsican populations (Supplementary Information). As Supplementary Table S1 illustrates, among the five classically used and biologically relevant measures of colouration, some are phenotypically correlated yet Spearman rank's correlation did not exceed 0.672 in absolute value. The strongest phenotypic correlation was between blue UV chroma and blue hue (Spearman rank's correlation ranging from -0.476 to -0.672 ). All other trait combinations showed correlations of absolute value less than 0.4 .

\section{Sexual dimorphism}

For each trait in both data sets, we measured the degree of sexual dimorphism in colour ornamentation by calculating a standardized effect size: Cohen's $d$, and its associated standard error (equations 10 and 16 in Nakagawa and Cuthill, 2007). Cohen's $d$ effect size is a dimensionless statistic; a value of 0.2 would typically be suggestive of small sexual dimorphism, whereas a value of 0.8 would be interpreted as revealing strong sexual dimorphism.

\section{Quantitative genetics}

Exploring fixed effects. Previous to conducting quantitative genetic models, we conducted linear mixed models to explore the contribution of fixed effects (year of measure, year of birth, period of measurement and individual age) to the various colour parameters in both data sets. For all traits, only year of
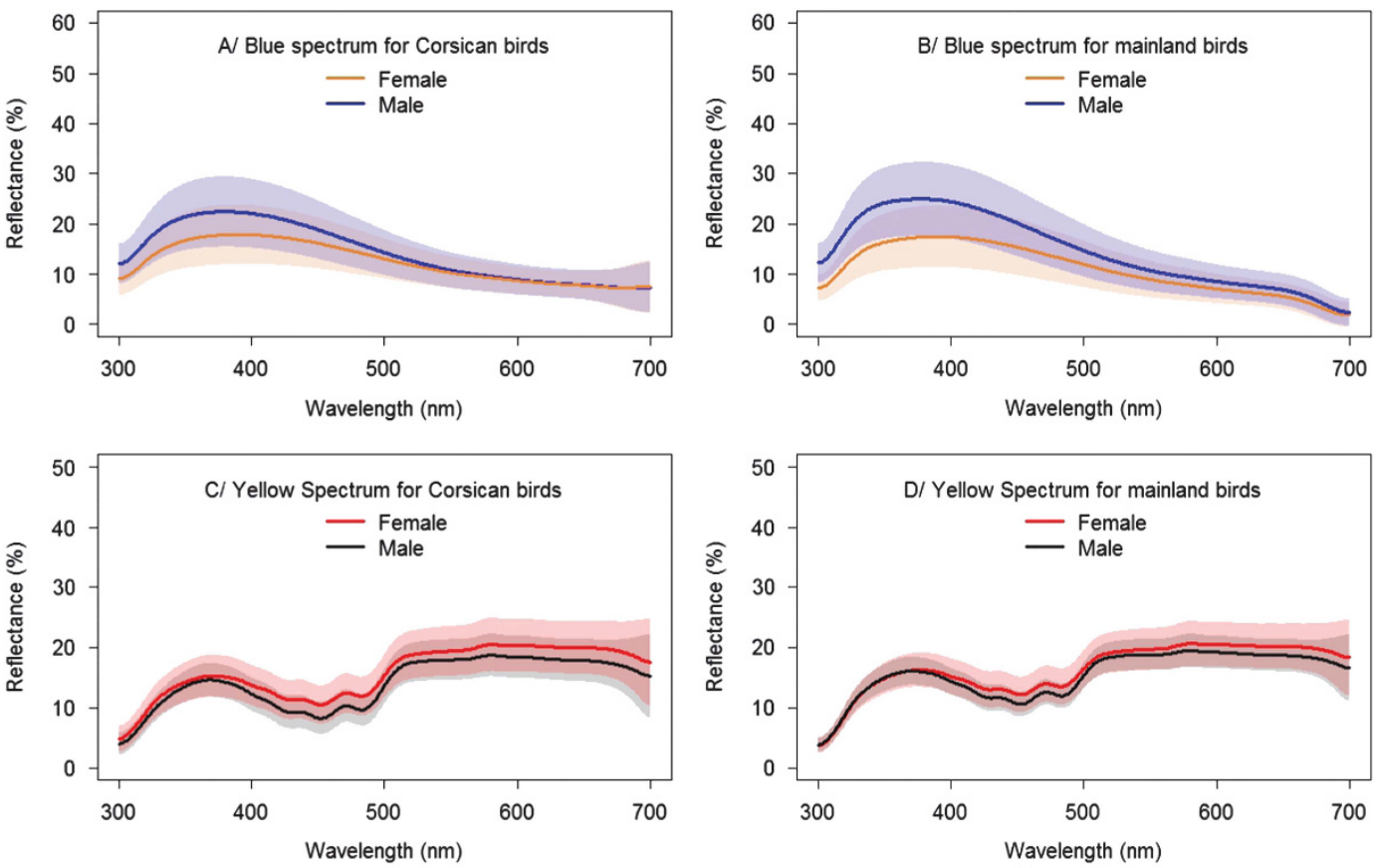

Figure 1 Average UV blue crown spectra for male (blue) and female (orange) blue tits sampled in 2011 (a) in Corsica and (b) on the mainland. Average yellow chest spectra for male (black) and female (red) blue tits sampled in 2011 (c) in Corsica and (d) on the mainland. Thick lines represent mean spectra and shaded areas associated s.d. values. Plots were realized using the R package 'pavo' (www.rafaelmaia.net/r-packages/pavo). 
Table 1 Sampling effort and mean values (with associated s.d. values) for colour traits measured in blue tit males and females in Rouvière (mainland) and in Corsica between 2005 and 2013

\begin{tabular}{|c|c|c|c|c|c|}
\hline & Blue brightness & Blue hue & Blue UV chroma & Yellow brightness & Yellow chroma \\
\hline \multicolumn{6}{|l|}{ Corsica, males } \\
\hline $\mathrm{Nb}$ measures & 882 & 882 & 882 & 870 & 958 \\
\hline Mean (s.d.) & $15.6(4.8)$ & 375.1 (11.7) & $0.39(0.04)$ & $16.1(3.7)$ & $0.80(0.18)$ \\
\hline \multicolumn{6}{|l|}{ Corsica, females } \\
\hline $\mathrm{Nb}$ measures & 865 & 865 & 865 & 825 & 930 \\
\hline Mean (s.d.) & $12.9(4.3)$ & $383.3(12.2)$ & $0.35(0.04)$ & $16.8(3.6)$ & $0.70(0.16)$ \\
\hline \multicolumn{6}{|l|}{ Rouvière, males } \\
\hline $\mathrm{Nb}$ measures & 810 & 810 & 810 & 769 & 769 \\
\hline Mean (s.d.) & $16.6(5.2)$ & 376.5 (11.3) & $0.38(0.03)$ & $17.0(3.5)$ & $0.63(0.17)$ \\
\hline \multicolumn{6}{|c|}{ Rouvière, females } \\
\hline $\mathrm{Nb}$ measures & 840 & 840 & 840 & 801 & 801 \\
\hline Mean (s.d.) & $14.2(5.4)$ & 388.1 (11.3) & $0.34(0.03)$ & $17.3(3.9)$ & $0.61(0.17)$ \\
\hline
\end{tabular}

Abbreviation: UV, ultraviolet.

Hue is in $\mathrm{nm}$.

measure was retained in all models as a categorical fixed effect (see details in Supplementary Information A1).

Univariate animal models. Genetic (co)variances, heritabilities and genetic correlations were estimated using restricted maximum-likelihood (REML) estimation procedures implemented in the software ASReml v3.0 (Gilmour et al., 2009). For each data set and colour measure, we first implemented a sex-specific univariate 'animal model' that combined the phenotypic measures for a given sex with the pedigree information to partition the phenotypic variance into an additive genetic variance $\left(V_{\mathrm{A}}\right)$, a variance due to permanent environment effects ( $V_{\mathrm{PE}}$, based on repeated observations of individuals) and a residual variance $\left(V_{\mathrm{R}}\right)$, while controlling for annual fluctuations using year as a single fixed effect. In such a model the phenotypic value of an individual $i$ is written as:

$$
y_{i}=\mu+Y E A R+a_{i}+P E_{i}+\varepsilon_{i}
$$

The additive genetic effect on individual $i\left(a_{i}\right)$, was assumed to be normally distributed with mean of zero and variance of $V_{\mathrm{A}}$. The permanent environment effect $\left(P E_{i}\right)$ and residual errors $\left(\varepsilon_{i}\right)$ were also assumed to be normally distributed, with zero means and variances $V_{\mathrm{PE}}$ and $V_{\mathrm{R}}$. Residual errors were assumed to be uncorrelated within individuals across measurements. In the Corsican model, a genetic group determined the Muro/Pirio origin for each bird.

The additive genetic variance estimates were tested against a null hypothesis of zero by carrying out likelihood ratio tests, where minus two times the difference in log likelihood between a model including the variance and a model without it was tested against the $\chi^{2}$ distribution with one degree of freedom.

Bivariate animal models and cross-sex additive genetic variance. In order to estimate the cross-sex additive genetic covariance for each colour measurement, we expanded Equation 1 to a bivariate model where the phenotypic values of males $\left(m_{i}\right)$ and of females $\left(f_{i}\right)$ are explained by fixed (year of measure) and random effects (as previously, additive genetic, permanent environment and residual effects):

$$
\begin{aligned}
& m_{i}=\mu_{m}+Y E A R_{m}+a_{m i}+P E_{m i}+\varepsilon_{m i} \\
& f_{i}=\mu_{f}+Y E A R_{f}+a_{f i}+P E_{f i}+\varepsilon_{f i}
\end{aligned}
$$

This bivariate animal model provides sex-specific estimations of additive genetic variances $\left(V_{A_{m}}, V_{A_{f}}\right)$, permanent environment variances $\left(V_{P E_{m}}, V_{P E_{f}}\right)$ and residual variances $\left(V_{R_{m}}, V_{R_{f}}\right)$. In this model, each character is sex specific and cannot be measured in males and females simultaneously, and hence this model cannot fit any between-individual (permanent environment) or within- individual (residual) covariance. However, it can fit a cross-sex additive genetic covariance $\left(\mathrm{COV}_{A_{m, f}}\right)$ from which we estimate the cross-sex additive genetic correlation:

$$
r_{A_{m, f}}=\frac{\operatorname{COV}_{A_{m, f}}}{\sqrt{V_{A_{m}} \times V_{A_{f}}}}
$$

A bivariate animal model was fitted for each colour trait in each population, with a genetic group specified for Muro and Pirio individuals in the case of the Corsican data set. The additive genetic covariance estimates were tested against a null hypothesis of zero by carrying out a likelihood ratio test using the $\chi^{2}$ distribution with one degree of freedom. In order to test for a genotype $\times$ sex interaction, which occurs when a given genotype has different phenotypic expressions in males versus females, we compared the original model with a model where $V_{A_{m}}=V_{A_{f}}=C O V_{A_{m f}}$ using likelihood ratio tests and the $\chi^{2}$ distribution with two degrees of freedom. To allow comparisons between traits and populations, we also report sex-specific coefficients of additive genetic variance $C V_{A_{m}}$ and $C V_{A_{f}}$ (Houle, 1992) in which the square root of the additive genetic variance is scaled by the trait mean:

$$
C V_{A}=100 \times \sqrt{V_{A}} / \bar{X}
$$

Including a Z-linked genetic variance. We conducted power analyses to determine the ability of the animal model to estimate sex-chromosomal and autosomal additive genetic variance given our blue tit pedigrees and data structures. Specifically, our goal was to determine whether we could detect Z-chromosome-linked additive genetic variance $\left(V_{Z}\right)$ in the blue tit colouration data. Our general approach was to use Monte Carlo simulation to reassign individual phenotypes with known (that is, simulated) sources of trait covariation in the population and then use animal models with each simulated data set to test the null hypothesis that Z-chromosomal additive genetic (co) variances were equal to zero. Over many replicate simulations, the proportion of significant $P$-values $(P<0.05)$ obtained from our null hypothesis tests reflect the power (the probability of rejecting the null hypothesis when it is false) of the animal model to estimate $V_{Z}$. We note that this does not determine the power of the animal model to provide unbiased estimates of autosomal $\left(V_{A}\right)$ and sex-linked $\left(V_{Z}\right)$ additive genetic variances (Supplementary Information).

We simulated random effects underlying observed phenotypes similar to those modeled for the observed data (Equation 2): additive genetic (autosomal and Z-linked), permanent environment and residual effects (Supplementary Information A3). We used 27 unique combinations of autosomal additive genetic, permanent environment and residual variances along with cross-sex autosomal and sex-linked additive genetic correlations (Supplementary 
Table S2). Within each of these unique combinations, the $Z$-linked additive genetic variance was set to one of seven values: $\sigma_{Z \text {-male }}{ }^{2}=1,10,30,50,60,70$ or 90 to assess the power at each level.

For each of the above parameter combinations, in each of the two data sets (Corsica and Rouvière), we simulated phenotypes for every individual (Supplementary Equation S1 in Supplementary Information) a total of 1000 different times. We used R (R Core Team, 2014) and the R package nadiv (Wolak, 2012) to simulate each of the above effects (Supplementary Information A3). We used the model of sex-chromosomal additive genetic variance of Fernando and Grossman (1990) that assumes no global sex chromosomal dosage compensation or recombination between the $\mathrm{Z}$ and $\mathrm{W}$ chromosomes.

Simulated phenotypes were analysed with an animal model implemented in the ASReml R package (v3.0, Butler et al., 2009). Models were conducted with and without the Z-linked additive genetic (co)variance terms, and minus two times the difference in these model log likelihoods was used to calculate a likelihood ratio test statistic. Probabilities of obtaining a difference in $\log$ likelihoods were assigned assuming an asymptotically $\chi^{2}$ distributed test statistic with three degrees of freedom. For a given set of parameters, we used the proportion of $P$-values $<0.05$ as an estimate of power. Full details are available in the Supplementary Information A3.

\section{RESULTS}

\section{Additive genetic (co)variances and heritabilities}

As detailed in Table 2, the bivariate animal models revealed that the chromatic part of the crown colouration (blue UV chroma and hue) was overall heritable (except for 2 of the 8 estimated colour parameters: male hue and female UV chroma in Corsica) with heritability estimates ranging from 0.07 to 0.19 in Corsica (0.73-4.06 for $\left.C V_{\mathrm{A}}\right)$ and from 0.18 to 0.23 in Rouvière (1.10-3.98 for $C V_{\mathrm{A}}$ ). In contrast, the achromatic part of the crown colouration (brightness) was heritable for both sexes (with heritabilities of 0.18 and 0.10 for males and females) in Corsica but not heritable for both sexes in the mainland Rouvière population (although $C V_{\mathrm{A}} \mathrm{s}$ were high), suggesting it is more sensitive to nongenetic variation than chromatic parameters. Similarly, the achromatic part of the yellow colouration was nonheritable in both sexes and populations, whereas the chromatic part was significantly heritable in males (heritabilities of 0.13 and 0.25 in Corsica and the mainland), but not in females.

Differences in model log likelihoods where sex-specific additive genetic variances- $V_{A_{m}}$ and $V_{A_{f}}$-were unconstrained or constrained to be equal indicated genotype by sex interactions only in two cases: in Corsica for blue hue $(P=0.003)$ and blue UV chroma $(P<0.0001)$. Estimated $\mathrm{COV}_{\mathrm{A}_{m, f}}$ (Table 2) for all blue measures and for Corsican yellow chroma were large and significantly greater than zero. $\mathrm{COV}_{\mathrm{A}_{m, f}}$ was not significantly different from zero for yellow chroma in Rouvière, yet this is most likely explained by the very small $V_{A_{f}}$ that prevents a correct estimation of the covariance.

Power analysis for sex-linked genetic variance

Overall, the power simulations revealed low power to estimate Z-linked additive genetic variance in our two data sets (see partial results in Figure 2 and Supplementary Information A3). Using a common rule of thumb for power, the Corsican data only achieve a minimum level of desired power (80\%) when the Z-linked betweensex additive genetic correlation is one (bottom row, Supplementary Figure S2a), Z-linked additive genetic variance is very high $(>70)$ and autosomal additive genetic variance is two. The animal model combined with the Rouvière population structure (Supplementary Figure S2b) achieves $80 \%$ power under less restrictive conditions, although this still requires Z-linked additive genetic variance to comprise at least $50 \%$ of total phenotypic variance (that is, $h^{2}$ Z-linked $\left.>0.5\right)$.

\section{Sexual colour dimorphism}

All colour traits displayed some sexual dimorphism, apart from yellow brightness in the Rouvière population (Figure 3, all paired one-sided Student's $t$-tests with $P<0.016$ except for yellow brightness in the Rouvière: $P=0.061$ ), with males being more colourful than females for both ornaments, with brighter blue and slightly brighter yellow.

\section{DISCUSSION}

Autosomal and sex-linked genetic variation for colour ornamentation in the blue tit

Autosomal genetic variation. Our quantitative genetic analyses reveal higher heritabilities for the crown blue UV colour than previously estimated (Hadfield et al., 2006a), confirm that the chromatic part of yellow colouration can be heritable in males (Evans and Sheldon, 2012) and reveal a lower heritability for yellow chroma in females than males.

Table 2 Heritability of colour features in male and female blue tits $\left(h_{m}^{2}\right.$ and $\left.h_{f}^{2}\right)$ and cross-sex additive genetic covariances $\left(\operatorname{COV}_{A_{m, f}}\right)$ and correlations $\left(r_{A_{m, f}}\right)$ estimated using bivariate animal models (with s.e. values)

\begin{tabular}{|c|c|c|c|c|c|c|c|c|c|}
\hline & $n b$ obs & $V_{A m}$ & $C V_{A_{m}}$ & $h_{m}^{2}$ & $V_{A_{f}}$ & $C V_{A_{f}}$ & $h_{f}^{2}$ & $\operatorname{COV}_{A_{m, f}}$ & $r_{A_{m, f}}$ \\
\hline \multicolumn{10}{|l|}{ Corsica } \\
\hline Blue brightness & 1795 & $3.73(1.02)$ & 12.34 & $0.18(0.05)$ & $1.59(0.76)$ & 9.81 & $0.10(0.05)$ & $2.37(1.30)$ & $0.97(0.54)$ \\
\hline Blue hue & 1795 & $7.48(4.98)$ & 0.73 & $0.07(0.04)$ & $13.59(6.18)$ & 0.96 & $0.11(0.05)$ & $10.06(7.98)$ & $1.00(0.87)$ \\
\hline Blue UV chroma & 1795 & $2.5 \mathrm{E} 10^{-4}\left(5.3 \mathrm{E} 10^{-5}\right)$ & 4.06 & $0.19(0.06)$ & $1.2 \mathrm{E} 10^{-4}\left(1.2 \mathrm{E} 10^{-4}\right)$ & 3.10 & $0.14(0.14)$ & $1.710^{-4}\left(7.9 \mathrm{E} 10^{-5}\right)$ & $0.99(0.68)$ \\
\hline Yellow brightness & 1772 & $0.95(0.61)$ & 6.05 & $0.07(0.05)$ & $0.73(0.60)$ & 5.07 & $0.06(0.05)$ & & \\
\hline Yellow chroma & 1957 & $3.6 \mathrm{E} 10^{-3}\left(1.2 \mathrm{E} 10^{-3}\right)$ & 7.56 & $0.13(0.04)$ & $3.6 \mathrm{E} 10^{-3}\left(2.7 \mathrm{E} 10^{-3}\right)$ & 8.51 & $0.15(0.11)$ & $3.6 \mathrm{E} 10^{-3}\left(1.7 \mathrm{E} 10^{-3}\right)$ & $1.00(0.63)$ \\
\hline \multicolumn{10}{|l|}{ Rouvière } \\
\hline Blue brightness & 1650 & 1.35 (1.05) & 7.01 & $0.06(0.05)$ & $1.33(1.27)$ & 8.15 & $0.07(0.07)$ & & \\
\hline Blue hue & 1650 & $17.24(7.48)$ & 1.10 & $0.18(0.07)$ & $21.37(5.58)$ & 1.19 & $0.20(0.05)$ & $19.04(5.65)$ & $0.99(0.28)$ \\
\hline Blue UV chroma & 1650 & $1.6 \mathrm{E} 10^{-4}\left(5.7 \mathrm{E} 10^{-5}\right)$ & 3.31 & $0.19(0.07)$ & $1.9 \mathrm{E} 10^{-4}\left(4.3 \mathrm{E} 10^{-5}\right)$ & 3.98 & $0.23(0.05)$ & $1.6 \mathrm{E} 10^{-4}\left(4.5 \mathrm{E} 10^{-5}\right)$ & $0.94(0.25)$ \\
\hline Yellow brightness & 1570 & $0.74(0.38)$ & 5.07 & $0.09(0.04)$ & $0.75(0.93)$ & 5.00 & $0.07(0.08)$ & & \\
\hline Yellow chroma & 1570 & $4.9 \mathrm{E} 10^{-3}\left(1.9 \mathrm{E} 10^{-3}\right)$ & 11.16 & $0.25(0.10)$ & $3.6 \mathrm{E} 10^{-3}\left(2.0 \mathrm{E} 10^{-3}\right)$ & 9.91 & $0.16(0.09)$ & $9.3 \mathrm{E} 10^{-4}\left(1.4 \mathrm{E} 10^{-3}\right)$ & $0.22(0.33)$ \\
\hline
\end{tabular}

Abbreviation: nb obs, number of measures for each trait; UV, ultraviolet.

The cross-sex additive genetic correlation is presented only for cases where at least one sex-specific additive genetic variance $\left(V_{A_{m}}, V_{A_{f}}\right)$ was significant. All significant results $(P<0.05)$ are in bold. Significance of $r_{A_{m}}$, was not assessed. 

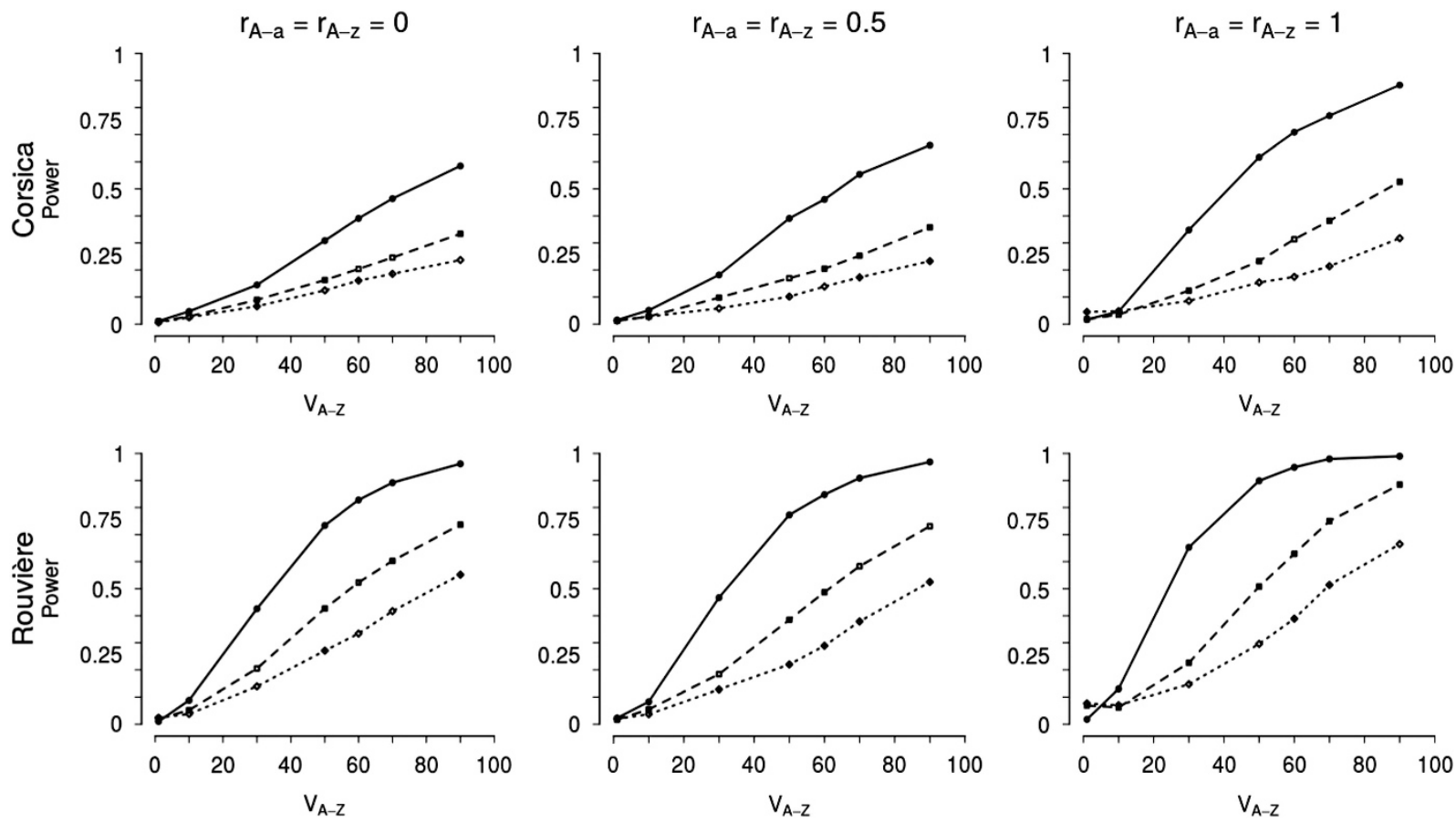

Figure 2 Power to estimate Z-chromosomal additive genetic variance in the Corsican (top row) and Rouvière (bottom row) populations. Between-sex additive genetic autosomal $\left(r_{A-a}\right)$ and Z-chromosomal $\left(r_{A-Z}\right)$ correlations vary from zero to one. Power is calculated as the proportion of simulations for which the model with Z-chromosomal additive genetic (co)variances fitted significantly better than a model without. Power was assessed at seven values of Z-chromosomal additive genetic variance (values along the $\mathrm{x}$ axes) and three values of autosomal additive genetic variance $\left(\mathrm{V}_{\mathrm{A}-\mathrm{a}}\right.$; solid $=2$, dashed $=50$, and dotted $=100$ lines).

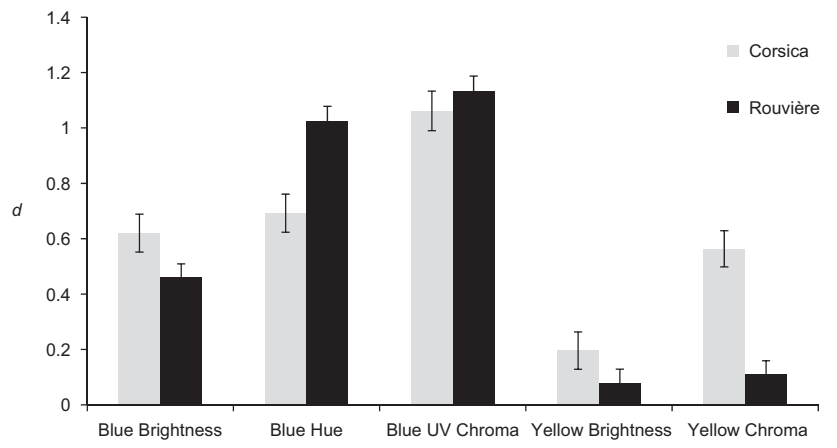

Figure 3 Sexual dimorphism (Cohen's d) on five colour traits in blue tits from Corsica (grey) and from the mainland (population of Rouvière, black). Bars represent s.e. values. See Table 1 for sample sizes and main text for statistics.

Blue UV colouration depends on the microstructure of the plumage (Prum, 2006), whereas yellow colouration is influenced by carotenoid contents (Partali et al., 1987) and by microstructure (Shawkey and Hill, 2005). Food is the sole source of carotenoids for blue tits as animals cannot synthesize them. Hence, stronger environmental dependence is expected in carotenoid-based colouration compared with the structurally based colouration. This prediction is upheld by our results for females, but not so much for males where there is no strong contrast between heritabilities for carotenoid-based and structurally based colouration.

The fact that male yellow chroma is as heritable as the UV blue colouration in both populations, and displays high $C V_{A_{m}}$, is very interesting. As yellow chroma is related to carotenoid content in the feathers, more chromatic individuals are often depicted as having higher foraging capacities and/or higher parasite resistance (see, for example, del Cerro et al., 2010). Our results suggest that more chromatic males have male offspring that are more chromatic themselves. This could be interpreted as more chromatic males having higher abilities at finding food and/or at parasite resistance, and that their male offspring inherit these aptitudes, either genetically or nongenetically. Indeed, although the additive genetic variance is estimated here based on a variety of relatedness types, the animal model cannot always decipher accurately between genetic versus shared environmental or social resemblance between relatives when the large majority of individuals in the pedigree are siblings or parentoffspring (Wolak and Keller, 2014). A male-specific social rather than genetic inheritance of yellow chroma, for example, mediated by paternal care, could explain why this trait is less heritable in females (although note that $C V_{\mathrm{A}} \mathrm{s}$ are of similar magnitude). As females disperse longer distances than males in the Blue tit (Matthysen et al., 2005), males sharing microhabitats could possibly lead to a malespecific nongenetic inheritance pattern. Such sex-specific environmental covariance between relatives needs to be investigated in future work, ideally using experimental approaches to isolate genetic and environmental effects. In any case, such father-son resemblance in yellow chroma makes it a good candidate for a sexually selected trait to optimize both direct and indirect benefits for females.

The moderate but significant heritabilities presented here are consistent with previous estimates in colour patches of blue tits (Hadfield and Owens, 2006) and great tits (Evans and Sheldon, 2012), yet they are much smaller than heritabilities associated with the sizes of melanin and white colour patches in other species (ranging from 0.28 to 0.90 , see, for example, Saino et al., 2013; Hubbard et al., 2015; 
Roulin and Jensen, 2015). Melanin and white patches have previously been suggested to be influenced by an individual's condition (Gustafsson et al., 1995; Griffith, 2000), including long-lasting effects of early environments (Roulin, 2016). However, our recent understanding on the genetic determinism for melanism (Theron et al., 2001; Ducrest et al., 2008), as well as the comparison with our present results suggest that variation in black and white ornaments may be less susceptible to body condition than structural and carotenoid-based colourations. In particular, we found that additive genetic variance explains only a small proportion of total variation in the achromatic part of yellow chest colouration, consistent with findings in other blue and great tit populations (Hadfield and Owens, 2006; Hadfield et al., 2006b; Drobniak et al., 2013). These results suggest that most variation in this aspect of colouration is likely attributable to environmental sources, including individual condition. Differences in condition dependence across colouration signals have been demonstrated experimentally using drug or nutritional treatments (McGraw et al., 2002; Hill et al., 2009). Two comparative analyses have also revealed that sexual dichromatism (used as a proxy of sexual selection) is more intense for carotenoid-based or structurally based colouration than for melanin-based colouration (Badyaev and Hill, 2000; Taysom et al., 2011).

Our variance partitioning in the blue UV crown colour reveals some striking differences between the two blue tit subspecies (for example, heritable blue brightness in Corsica only), although the absence of other comparable studies prevents any generalization. In addition, we found higher heritabilities overall than Hadfield et al. (2006a), thereby illustrating that the genetic determinism of colouration can vary across populations and requires further quantitative genetic investigations of colouration both within and across species (see review in Mundy, 2006).

Sex-linked genetic variation. Although it is now clear that many genes underlying sexual dimorphism are not sex linked (Badyaev, 2002) and that sex linkage is not a requirement for sexual dimorphism (Fairbairn and Roff, 2006; Dean and Mank, 2014; Roulin and Jensen, 2015), there is accumulating evidence for sex linkage of genes underlying sexually dimorphic traits, especially with the increasing accessibility of genetic mapping in nonmodel organisms (Charlesworth and Mank, 2010; Huang and Rabosky, 2015). Recent evidence suggests that Z-linked genetic variance can explain as much as $40 \%$ of the total phenotypic variation in colour ornaments of birds (see Introduction and Husby et al., 2013). However, the statistical power to estimate Z-linked additive genetic variance in our two data sets was very low (see partial results in Figure 2 and Supplementary Information A3). Although we found more power in Rouvière than the Corsican populations (possibly because of a higher pedigree connectedness), it is unlikely, however, that an animal model using data collected from either population would have enough power to detect Z-linked additive genetic variance. Only when the simulated autosomal additive genetic variance was at its lowest value and the simulated Z-chromosomal additive genetic variance was among its highest values would conventional rules of thumb deem there to be sufficient power (that is, power $>80 \%$ ) to calculate Z-chromosomal additive genetic variance. Although empirical estimates of sex-chromosomal additive genetic variance are few, it seems an unlikely condition to find such high sexchromosomal heritability almost at the exclusion of autosomal heritability. Overall, these simulations revealed that we could not test the hypotheses involving sex-linked genetic variation, and that most if not all previously published results on sex-linked genetic variance suffered from similar lack of power. This is a worrying report that calls for further simulations to determine the structure and size of pedigree and data required to estimate sex-linked genetic variance.

\section{Cross-sex genetic covariances and female ornamentation}

In the animal kingdom, dimorphic traits under sexual selection have been shown to be associated with a whole range of cross-sex genetic correlations : from low (for example, in Drosophila serrata; Chenoweth and Blows, 2003) to very strong correlations (for example, in the red deer Cervus elaphus; Pavitt et al., 2014). The sparse and contrasted results prevent from drawing general conclusions on the link between genetic covariances across sexes and the evolution of sexual traits. In our study, estimated cross-sex additive genetic correlation- $r_{A_{m, f}}-$ were high (close to one), even in cases where the trait was not significantly heritable in one sex (for example, blue hue and yellow chroma in Corsica, see Table 2 for details). To our knowledge, only one other study explored $r_{A_{m, f}}$ for blue structural colours (in Florida scrub-jays, Tringali et al., 2015), with similar results of very strong cross-sex genetic correlations. These results validate the fundamental assumption underlying the correlated response hypothesis (Lande, 1980). They suggest that evolution of female crown colouration could be drastically constrained by indirect selection acting on males.

Analogous conclusions can be drawn from estimates of $r_{A_{m, f}}$ in carotenoid-based ornaments: the evolution of colouration in one sex is likely to have a strong influence on the colouration in the other sex. However, we found large variability in our estimates, with $r_{A_{m, f}}$ of yellow chest chroma ranging from 0.22 in Rouvière to 1 in Corsica. The few estimates of cross-sex genetic correlations for carotenoidbased colour traits so far in the literature show a similarly large range of values for $r_{A_{m, f}}$. High additive genetic correlation was found for beak redness in the zebra finch $\left(r_{A_{m, f}}=0.926\right.$; Schielzeth et al., 2012) but a study of yellow brightness, saturation and hue in blue tit nestlings showed $r_{A_{m f}}$ ranging from -0.13 to 0.19 with very large confidence intervals (Drobniak et al., 2013). These very divergent results call for further investigations on cross-sex genetic correlations in carotenoid-based ornaments in a wider range of species and populations. New genomic tools might also soon allow the identification of genomic regions involved in colour variation in both males and females, thereby revealing whether the same genes influence plumage colouration in both sexes (Roulin and Ducrest, 2013; Kraaijeveld, 2014; Huang and Rabosky, 2015).

Our quantitative genetic analyses used social pedigrees that were only partially corrected for extra-pair paternity. Hence, additive genetic variances and heritabilities in Table 2 could be underestimated, although as said above they were overall larger than reported in previous studies (Charmantier and Réale, 2005). Unfortunately, little is known on how errors in paternity assignment due to extra-pair reproduction can affect the estimation of genetic covariances and sex-linked genetic variance (Reid, 2014). A study combining data on extra-pair occurrence and parental colour is planned for our study populations so that we may quantify to what extent missassigned paternity will bias quantitative genetic (co)variance estimates.

\section{Linking the degree of sexual dimorphism to cross-sex additive genetic covariance}

In accordance with previous studies in this species (Andersson et al., 1998; Hunt et al., 1998; Delhey and Peters, 2008; Doutrelant et al., 2008) blue characteristics were all highly dimorphic, with the strongest dimorphism expressed in the blue UV chroma, whereas yellow characteristics showed small or moderate dimorphism. Interestingly, Corsican subspecies of blue tits were significantly more dimorphic for yellow chroma than mainland birds (two-sided Student's 
t-test, $P=4 \times 10^{-8}$ ), whereas the reverse was true for blue hue (twosided $t$-test, $P=0.0001)$. Although sexual dimorphism in yellow is usually considered very small for this species and possibly below the detectable level for birds (Delhey et al., 2010), strong dichromatism has been reported once before, in central Spain (Garcia-Navas et al., 2012). Our personal observations across the ultramarinus complex (C Doutrelant and G Sorci, unpublished data) suggest that the yellow sexual dimorphism might be a characteristic of blue tits in the southern part of the species distribution. These observations limited to the southern edge of the distribution could be explained by differences in selective forces acting on this ornament. Southern blue tit populations are subject to more drastic food limitation than northern ones. Comparative selection analyses would confirm whether these increased environmental constraints result in different selection pressures acting on male and/or female yellow colouration, in particular on yellow chroma, as it is directly linked to the carotenoid content of the feather, and is heritable.

Homologous characters in the two sexes, such as blue crown colour and yellow chest colour in blue tits, are presumably controlled, at least in the early evolution of these traits, by very similar sets of genes, leading to strong cross-sex genetic covariance. As any dimorphic character, these traits are likely to be under antagonistic selection in males and females (Rice, 1984) that, combined with a strong cross-sex genetic covariance, would create an intralocus sexual conflict (Lande, 1980; Bonduriansky and Rowe, 2005; Poissant et al., 2010). This leads to the classic prediction that the degree of sexual dimorphism should be inversely correlated with the level of $\mathrm{COV}_{\mathrm{A}_{\mathrm{m}, \mathrm{f}}}$ (Fairbairn and Roff, 2006) and of $\mathrm{r}_{\mathrm{A}_{m, f}}$ (Lande, 1980; Bonduriansky and Rowe, 2005). The negative relationship between the cross-sex additive genetic covariance and the magnitude of sexual dimorphism is generally upheld over a range of trait types and across a variety of animal and plant species (Fairbairn and Roff, 2006; Bonduriansky, 2007; Poissant et al., 2010). However, studies on the role of ornamentation in sexual selection have largely focussed on conspicuously sexually dimorphic species, neglecting species with low or no sexual dimorphism (see reviews in Kraaijeveld et al., 2007; Poissant et al., 2010). Estimating cross-sex genetic covariances for weakly dimorphic or nondimorphic species/ traits is now a necessary stepping stone in our understanding of the evolution of sexual dimorphism (Lande, 1980). In our blue tit study, this prediction was not validated when comparing the five colour traits with varying degrees of dimorphism. Indeed, the most dimorphic traits (blue UV chroma and blue UV hue) displayed strong $C O V_{A_{m, f}}$ in both data sets, with $r_{A_{m, f}}$ close to 1 , and the only nonsignificant $C O V_{A_{m, f}}$ was found in one of the least dimorphic traits (yellow chroma). These results imply that the evolution of sexual dimorphism in this species was not facilitated by low intersexual genetic covariance, suggesting other mechanisms should be considered.

First, the observed sexual dimorphism in colour could be driven by environmental differences rather than genetic ones, with a greater sensitivity of one sex to environmental variation. For instance, it has been shown in insects that sex-specific phenotypic plasticity can generate variation in sexual size dimorphism (Stillwell et al., 2010). Differences in plasticity between males and females should lead to consistent differences in sex-specific heritabilities for similar levels of $C V_{\mathrm{A}}$ (Houle, 1992), but this is not a general result witnessed across the focal traits in Table 2. Second, genes linked to sex chromosomes could explain the sexual dimorphism over and above the autosomal genetic (co)variances estimated here, although we could not estimate such sex-linked genetic variance. Third, cross-sex genetic covariances may have changed over the course of the evolution of sexual dimorphism. Meagher (1992) has suggested that during the evolution of sexual dimorphism, loci that show sex-specific expression should be strongly selected for and should become fixed, thereby no longer contributing to the additive genetic variance. This could explain how $C O V_{A_{m, f}}$ could be temporarily low or negative during the evolution of dimorphism, but then large and positive once the sex-specific loci are fixed.

An important limitation of our study is that we could not adopt a truly multivariate approach where genetic covariances between suites of traits within and between the sexes might provide a different view on the genetic constraints for the evolution of sexual dimorphism. Indeed, the evolutionary trajectory of a given sex-specific character can be constrained or facilitated by selection acting on the variance displayed by the same trait expressed in the other sex, but also by positive or negative genetic correlations with other traits within and between both sexes (Blows and Hoffmann, 2005; Poissant et al., 2010). For this reason, future studies will need to integrate cross-sex genetic covariances across traits with multivariate selection analyses (Lande, 1980; Chenoweth et al., 2010) in order to fully uncover how sexual antagonistic selection and intralocus sexual conflicts can promote or constrain the evolution of divergent male and female traits (Wyman et al., 2013). Such an approach has been adopted recently in a study of a laboratory population of Drosophila melanogaster (Ingleby et al., 2014) and also in a natural population of barn owl (Roulin and Jensen, 2015). Yet, model complexity combined with data availability still largely prevent such multivariate analyses in many natural populations.

\section{CONCLUSION}

Overall, this study brought three major advancements in our understanding of the evolution of colour ornamentation and sexual dimorphism. First, the present analyses demonstrated heritability for UV colouration (in both sexes) and yellow colouration (in males), a major requirement for the evolution of colour through sexual or social selection. Second, our simulations revealed the low power of animal models to estimate sex-linked additive genetic variance in wild populations, thereby hampering our ability to test a major hypothesis for the evolution of sexual dimorphism. Third, in the current debate on the evolution of female ornaments, the present results suggest that cross-sex genetic correlations can be very high in colour traits across varying degrees of dimorphism. A fine-scale analysis of sex-specific forces of natural, social and sexual selection is now required to determine the role of indirect (selection acting on males) and direct selection for the evolution of female ornaments. Future genomic studies should be used to determine whether the same genes underlie colouration in males and females.

\section{DATA ARCHIVING}

Phenotypic and pedigree data sets available from the Dryad Digital Repository: http://dx.doi.org/10.5061/dryad.gp384. The raw data will be embargoed for 5 years, but could be made available during this period upon request to the authors.

\section{CONFLICT OF INTEREST}

The authors declare no conflict of interest.

\section{ACKNOWLEDGEMENTS}

We thank all the people who collected feathers in the field and participated to the blue tit long-term program, in particular P Perret, M Lambrechts, J Blondel, D Réale, D Garant, M Porlier, G Dubuc Meissier, P Marrot, P Giovanini and many field assistants. We thank all the people who performed the colour measures, and in particular Emeline Mourocq, Afiwa Midamegbe and Maria 
del Rey. We thank Arild Husby for precious help with ASReml scripts, Alastair Wilson and the WAMBAM attendees for further discussions on the analyses, Mandy Thion for preliminary analyses, Alexandre Roulin and two anonymous reviewers for comments on the manuscript. The European Research Council (Starting Grant ERC-2013-StG-337365-SHE to AC), the French Agence Nationale de la Recherche (Grant ANR-12-ADAP-0006-02-PEPS to AC; ANR 09-JCJC-0050-0 to CD), the Région Languedoc Roussillon and the OSU-OREME provided funding. Bird capture, ringing and measurements were performed under individual ringing permits delivered by the CRBPO, with approval from the Animal Care and Use Committee Languedoc-Roussillon (CEEA-LR-12066) and with a Regional authorization for capture delivered by the Prefet de Corse (Arrêté no. 2012167-0003).

Alonso-Alvarez C, Doutrelant C, Sorci G (2004). Ultraviolet reflectance affects male-male interactions in the blue tit (Parus caeruleus ultramarinus). Behav Ecol 15: 805-809. Amundsen T (2000). Why are female birds ornamented? Trends Ecol Evol 15: 149-155. Andersson M (1994). Sexual Selection. Princeton University Press: Princeton, NJ, USA. Andersson S, Ornborg J, Andersson M (1998). Ultraviolet sexual dimorphism and assortative mating in blue tits. Proc $R$ Soc Lond B 265: 445-450.

Badyaev AV (2002). Growing apart: an ontogenetic perspective on the evolution of sexual size dimorphism. Trends Ecol Evol 17: 369-378.

Badyaev AV, Hill GE (2000). Evolution of sexual dichromatism: contribution of carotenoidversus melanin-based coloration. Biol J Linn Soc 69: 153-172.

Blondel J, Thomas DW, Charmantier A, Perret P, Bourgault P, Lambrechts MM (2006) A thirty-year study of phenotypic and genetic variation of blue tits in Mediterranean habitat mosaics. Bioscience 56: 661-673.

Blows MW, Hoffmann AA (2005). A reassessment of genetic limits to evolutionary change. Ecology 86: 1371-1384.

Bonduriansky $R$ (2007). The genetic architecture of sexual dimorphism: the potential roles of genomic imprinting and condition dependence. In: Fairbairn DJ, Blanckenhorn WU, Szekely T (eds) Sex, Size And Gender Roles: Evolutionary Studies of Sexual Size Dimorphism. Oxford University Press: Oxford, UK, pp 176-185.

Bonduriansky R, Rowe L (2005). Intralocus sexual conflict and the genetic architecture of sexually dimorphic traits in Prochyliza xanthostoma (Diptera : Piophilidae). Evolution 59: 1965-1975.

Butler DG, Cullis BR, Gilmour AR, Gogel BJ (2009). ASReml-R Reference Manual. Series title, Queensland Department of Primary Industries. Available at: http://www.vsni.co.uk/ software/asreml/.

Byrne PG, Rice WR (2006). Evidence for adaptive male mate choice in the fruit fly Drosophila melanogaster. Proc R Soc Lond B 273: 917-922.

Charlesworth D, Mank JE (2010). The birds and the bees and the flowers and the trees: lessons from genetic mapping of sex determination in plants and animals. Genetics 186: 9-31.

Charmantier A, Blondel J, Perret P, Lambrechts MM (2004). Do extra-pair paternities provide genetic benefits for female blue tits (Parus caeruleus)? J Avian Biol 35: 524-532.

Charmantier A, Doutrelant C, Dubuc Messier G, Fargevieille A, Szulkin M (2016). Mediterranean blue tits as a case study of local adaptation. Evol App/ 9: 135-152.

Charmantier A, Garant D, Kruuk LEB (eds) (2014). Quantitative Genetics in the Wild. Oxford University Press: Oxford, UK, p 304.

Charmantier A, Perret P (2004). Manipulation of nest-box density affects extra-pair paternity in a population of blue tits (Parus caeruleus). Behav Ecol Sociobiol 56 360-365.

Charmantier A, Réale D (2005). How do misassigned paternities affect the estimation of heritability in the wild? Mol Ecol 14: 2839-2850.

Chenoweth SF, Blows MW (2003). Signal trait sexual dimorphism and mutual sexual selection in Drosophila serrata. Evolution 57: 2326-2334.

Chenoweth SF, Rundle HD, Blows MW (2010). The contribution of selection and genetic constraints to phenotypic divergence. Am Nat 175: 186-196.

Clutton-Brock T (2007). Sexual selection in males and females. Science 318: 1882-1885

Dale J, Dey CJ, Delhey K, Kempenaers B, Valcu M (2015). The effects of life history and sexual selection on male and female plumage colouration. Nature 527: 367-370.

Darwin C (1871). The Descent of Man, and Selection in Relation to Sex. John Murray: London.

Dean R, Mank JE (2014). The role of sex chromosomes in sexual dimorphism: discordance between molecular and phenotypic data. J Evol Biol 27: 1443-1453.

del Cerro S, Merino S, Martinez-de la Puente J, Lobato E, Ruiz-de-Castaneda R, Rivero-de Aguilar J et al. (2010). Carotenoid-based plumage colouration is associated with blood parasite richness and stress protein levels in blue tits (Cyanistes caeruleus). Oecologia 162: 825-835.

Delhey K, Peters A (2008). Quantifying variability of avian colours: are signalling traits more variable? PLoS One 3: e1689.

Delhey K, Roberts ML, Peters A (2010). The carotenoid-continuum: carotenoid-based plumage ranges from conspicuous to cryptic and back again. BMC Ecol 10: 13 .

Dijkstra PD, Seehausen O, Groothuis TGG (2008). Intrasexual competition among females and the stabilization of a conspicuous colour polymorphism in a Lake Victoria cichlid fish. Proc $R$ Soc Lond B 275: 519-526.
Doutrelant C, Gregoire A, Grnac N, Gomez D, Lambrechts MM, Perret P (2008). Female coloration indicates female reproductive capacity in blue tits. J Evol Biol 21: 226-233.

Doutrelant C, Gregoire A, Midamegbe A, Lambrechts M, Perret P (2012). Female plumage coloration is sensitive to the cost of reproduction. An experiment in blue tits. J Anim Ecol 81: 87-96.

Dreiss A, Richard M, Moyen F, White J, Moller AP, Danchin E (2006). Sex ratio and male sexual characters in a population of blue tits, Parus caeruleus. Behav Ecol 17: 13-19.

Drobniak SM, Wiejaczka D, Arct A, Dubiec A, Gustafsson L, Cichon M (2013). Low crosssex genetic correlation in carotenoid-based plumage traits in the blue tit nestlings (Cyanistes caeruleus). PLoS One 8: e69786.

Ducrest AL, Keller L, Roulin A (2008). Pleiotropy in the melanocortin system, coloration and behavioural syndromes. Trends Ecol Evol 23: 502-510.

Evans SR, Schielzeth H, Forstmeier W, Sheldon BC, Husby A (2014). Nonautosomal genetic variation in carotenoid coloration. Am Nat 184: 374-383.

Evans SR, Sheldon BC (2012). Quantitative genetics of a carotenoid-based color: heritability and persistent natal environmental effects in the great tit. Am Nat 179 : 79-94.

Fairbairn DJ, Roff DA (2006). The quantitative genetics of sexual dimorphism: assessing the importance of sex-linkage. Heredity 97: 319-328.

Fernando RL, Grossman M (1990). Genetic evaluation with autosomal and X-chromosomal inheritance. Theor Appl Genet 80: 75-80.

Garcia-Navas V, Ferrer ES, Sanz JJ (2012). Plumage yellowness predicts foraging ability in the blue tit Cyanistes caeruleus. Biol J Linn Soc 106: 418-429.

Gilmour AR, Gogel BJ, Cullis BR, Thompson R (2009). ASReml User Guide Release 3.0. VSN International Ltd: Hemel Hempstead, HP1 1ES, UK.

Gomez D (2006). AVICOL, a program to analyse spectrometric data. Last update October 2011. Free executable available at http://sites.google.com/site/avicolprogram/ or from the author at dodogomez@yahoo.fr.

Griffith SC (2000). A trade-off between reproduction and a condition-dependent sexually selected ornament in the house sparrow Passer domesticus. Proc $R$ Soc Lond B 267: 1115-1119.

Griggio M, Valera F, Casas A, Pilastro A (2005). Males prefer ornamented females: a field experiment of male choice in the rock sparrow. Anim Behav 69: 1243-1250.

Gustafsson L, Qvarnstrom A, Sheldon BC (1995). Trade-offs between life-history traits and a secondary sexual character in male collared flycatchers. Nature 375: 311-313.

Hadfield JD, Burgess MD, Lord A, Phillimore AB, Clegg SM, Owens IPF (2006a). Direct versus indirect sexual selection: genetic basis of colour, size and recruitment in a wild bird. Proc R Soc Lond B 273: 1347-1353.

Hadfield JD, Nutall A, Osorio D, Owens IPF (2007). Testing the phenotypic gambit: phenotypic, genetic and environmental correlations of colour. J Evol Biol 20: 549-557.

Hadfield JD, Owens IPF (2006). Strong environmental determination of a carotenoid-based plumage trait is not mediated by carotenoid availability. J Evol Biol 19: 1104-1114.

Hadfield JD, Richardson DS, Burke T (2006b). Towards unbiased parentage assignment: combining genetic, behavioural and spatial data in a Bayesian framework. $\mathrm{Mol} E \mathrm{Eol} 15$ 3715-3730.

Heinsohn R, Legge S, Endler JA (2005). Extreme reversed sexual dichromatism in a bird without sex role reversal. Science 309: 617-619.

Hill GE, Hood WR, Huggins K (2009). A multifactorial test of the effects of carotenoid access, food intake and parasite load on the production of ornamental feathers and bil coloration in American goldfinches. J Exp Biol 212: 1225-1233.

Hill GE, McGraw KJ (eds) (2006). Bird Coloration, Vol. I: Mechanisms, Function, and Evolution. Harvard University Press: Boston, MA, USA, p 640.

Houle D (1992). Comparing evolvability and variability of quantitative traits. Genetics 130 : 195-204.

Huang H, Rabosky DL (2015). Sex-linked genomic variation and its relationship to avian plumage dichromatism and sexual selection. BMC Evol Biol 15: 199.

Hubbard JK, Jenkins BR, Safran RJ (2015). Quantitative genetics of plumage color: lifetime effects of early nest environment on a colorful sexual signal. Ecol Evol 5: 3436-3449.

Hunt S, Bennett ATD, Cuthill IC, Griffiths R (1998). Blue tits are ultraviolet tits. Proc R Soc Lond B 265: 451-455.

Hunt S, Cuthill IC, Bennett ATD, Griffiths R (1999). Preferences for ultraviolet partners in the blue tit. Anim Behav 58: 809-815.

Husby A, Schielzeth H, Forstmeier W, Gustafsson L, Qvarnstrom A (2013). Sex chromosome linked genetic variance and the evolution of sexual dimorphism of quantitative traits. Evolution 67: 609-619.

Ingleby FC, Innocenti P, Rundle HD, Morrow EH (2014). Between-sex genetic covariance constrains the evolution of sexual dimorphism in Drosophila melanogaster. J Evol Biol 27: 1721-1732.

Isaksson C, Ornborg J, Prager M, Andersson S (2008). Sex and age differences in reflectance and biochemistry of carotenoid-based colour variation in the great tit Parus major. Biol J Linn Soc 95: 758-765.

Johnsen A, Delhey K, Andersson S, Kempenaers B (2003). Plumage colour in nestling blue tits: sexual dichromatism, condition dependence and genetic effects. Proc $R$ Soc Lond B 270: 1263-1270.

Kingma SA, Komdeur J, Vedder O, von Engelhardt N, Korsten P, Groothuis TGG (2009). Manipulation of male attractiveness induces rapid changes in avian maternal yolk androgen deposition. Behav Ecol 20: 172-179.

Kraaijeveld K (2014). Reversible trait loss: the genetic architecture of female ornaments. In: Futuyma DJ (ed) Annual Review of Ecology, Evolution, and Systematics, Vol 45. Annual Reviews: Palo Alto, pp 159-177.

Kraaijeveld K, Kraaijeveld-Smit FJL, Komdeur J (2007). The evolution of mutua ornamentation. Anim Behav 74: 657-677. 
Lande R (1980). Sexual dimorphism, sexual selection, and adaption in polygenic characters. Evolution 34: 292-305.

Larsen CT, Holand AM, Jensen H, Steinsland I, Roulin A (2014). On estimation and identifiability issues of sex- linked inheritance with a case study of pigmentation in Swiss barn owl (Tyto alba). Ecol Evol 4: 1555-1566.

Lebas NR (2006). Female finery is not for males. Trends Ecol Evol 21: 170-173.

Limbourg T, Mateman AC, Andersson S, Lessells CM (2004). Female blue tits adjust parental effort to manipulated male UV attractiveness. Proc $R$ Soc Lond $B$ 271: 1903-1908.

Limbourg T, Mateman AC, Lessells CM (2013). Opposite differential allocation by males and females of the same species. Biol Lett 9: 20120835.

Matthysen E, Van de Casteele T, Adriaensen F (2005). Do sibling tits (Parus major, $P$. caeruleus) disperse over similar distances and in similar directions? Oecologia 143: 301-307.

McGraw KJ, Mackillop EA, Dale J, Hauber ME (2002). Different colors reveal different information: how nutritional stress affects the expression of melanin- and structurally based ornamental plumage. J Exp Biol 205: 3747-3755.

Meagher TR (1992). The quantitative genetics of sexual dimorphism in Silene Latifolia (Caryophyllaceae). 1. Genetic variation. Evolution 46: 445-457.

Midamegbe A, Gregoire A, Staszewski V, Perret P, Lambrechts MM, Boulinier T et al. (2013). Female blue tits with brighter yellow chests transfer more carotenoids to their eggs after an immune challenge. Oecologia 173: 387-397.

Mundy NI (2006). Genetic basis of color variation in wild birds. In: Hill GE, McGraw KJ (eds) Bird coloration. Vol. 1. Mechanisms and measurements. Harvard University Press: Cambridge, MA, USA, pp 469-506.

Nakagawa S, Cuthill IC (2007). Effect size, confidence interval and statistical significance: a practical guide for biologists. Biol Rev 82: 591-605.

Parker TH (2013). What do we really know about the signalling role of plumage colour in blue tits? A case study of impediments to progress in evolutionary biology. Biol Rev $\mathbf{8 8}$ 511-536.

Parker TH, Wilkin TA, Barr IR, Sheldon BC, Rowe L, Griffith SC (2011). Fecundity selection on ornamental plumage colour differs between ages and sexes and varies over small spatial scales. J Evol Biol 24: 1584-1597.

Partali V, Liaaen-Jensen S, Slagsvold T, Lifjeld JT (1987). Carotenoids in food chain studies-II. The food chain of Parus SPP. Monitored by carotenoid analysis. Comp Biochem Physiol B 87: 885-888.

Pavitt AT, Walling CA, Pemberton JM, Kruuk LEB (2014). Heritability and cross-sex genetic correlations of early-life circulating testosterone levels in a wild mammal. Biol Lett 10: 4 .

Peters A, Kurvers RHJM, Roberts ML, Delhey K (2011). No evidence for general conditiondependence of structural plumage colour in blue tits: an experiment. J Evol Biol 24: 976-987.

Poissant J, Wilson AJ, Coltman DW (2010). Sex-specific genetic variance and the evolution of sexual dimorphism: a systematic review of cross-sex genetic correlations. Evolution 64: 97-107.

Price DK (1996). Sexual selection, selection load and quantitative genetics of zebra finch bill colour. Proc R Soc Lond B 263: 217-221.

Price DK, Burley NT (1993). Constraints on the evolution of attractive traits: Genetic (co) variance of Zebra Finch bill color. Heredity 71: 405-412.

Prum RO (2006). Anatomy, physics and evolution of avian structural colors. In: Hill GE, McGraw KJ (eds) Bird Coloration, Vol. I: Mechanisms, Function, and Evolution. Harvad University Press: Boston, MA, USA, pp 295-353.

$\mathrm{R}$ Core Team (2014). R: A Language and Environment for Statistical Computing. R Foundation for Statistical Computing: Vienna, Austria.

Reid JM (2014). Quantitative genetic approaches to understanding sexual selection and mating system evolution in the wild. In: Charmantier A, Garant D, Kruuk LEB (eds) Quantitative Genetics in the Wild. Oxford University Press: Oxford, UK, pp 34-53.

Rémy A, Gregoire A, Perret P, Doutrelant C (2010). Mediating male-male interactions: the role of the UV blue crest coloration in blue tits. Behav Ecol Sociobiol 64: 1839-1847.

Rice WR (1984). Sex chromosomes and the evolution of sexual dimorphism. Evolution 38 735-742.
Roulin A (2016). Condition-dependence, pleiotropy and the handicap principle of sexual selection in melanin-based colouration. Biol Rev 91: 328-348.

Roulin A, Ducrest AL (2013). Genetics of colouration in birds. Semin Cell Dev Biol 24: 594-608.

Roulin A, Jensen H (2015). Sex-linked inheritance, genetic correlations and sexual dimorphism in three melanin-based colour traits in the barn owl. J Evol Biol 28: 655-666.

Saino N, Romano M, Rubolini D, Teplitsky C, Ambrosini R, Caprioli M et al. (2013). Sexual dimorphism in melanin pigmentation, feather coloration and its heritability in the barn swallow (Hirundo rustica). PLoS One 8: e58024.

Savalli UM (2001). Sexual selection. In: Fox CW, Roff DA, Fairbairn DJ (eds) Evolutionary Ecology - Concepts and Case Studies. Oxford University Press: New York, pp 207-221.

Schielzeth H, Kempenaers B, Ellegren H, Forstmeier W (2012). QTL linkage mapping of zebbra finch beak color shows an oligogenic control of a sexually selected trait. Evolution 66: 18-30.

Shawkey MD, Hill GE (2005). Carotenoids need structural colours to shine. Biol Lett 1: 121-124.

Sheldon BC, Andersson S, Griffith SC, Ornborg J, Sendecka J (1999). Ultraviolet colour variation influences blue tit sex ratios. Nature 402: 874-877.

Stillwell RC, Blanckenhorn WU, Teder T, Davidowitz G, Fox CW (2010). Sex differences in phenotypic plasticity affect variation in sexual size dimorphism in insects: from physiology to evolution. Annu Rev Entomol 55: 227-245.

Svensson PA, Wong BBM (2011). Carotenoid-based signals in behavioural ecology: a review. Behaviour 148: 131-189.

Taysom AJ, Stuart-Fox D, Cardoso GC (2011). The contribution of structural-, psittacofulvin- and melanin-based colouration to sexual dichromatism in Australasian parrots. $J$ Evol Biol 24: 303-313.

Theron E, Hawkins K, Bermingham E, Ricklefs RE, Mundy NI (2001). The molecular basis of an avian plumage polymorphism in the wild: a melanocortin-1-receptor point mutation is perfectly associated with the melanic plumage morph of the bananaquit, Coereba flaveola. Curr Biol 11: 550-557.

Tobias JA, Montgomerie R, Lyon BE (2012). The evolution of female ornaments and weaponry: social selection, sexual selection and ecological competition. Philos Trans $R$ Soc Lond B 367: 2274-2293.

Tringali A, Bowman R, Husby A (2015). Selection and inheritance of sexually dimorphic juvenile plumage coloration. Ecol Evol 5: 5413-5422.

Vedder O, Korsten P, Magrath MJL, Komdeur J (2008). Ultraviolet plumage does not signal social status in free-living blue tits; an experimental test. Behav Ecol 19: 410-416.

Wolak ME (2012). nadiv: an R package to create relatedness matrices for estimating nonadditive genetic variances in animal models. Methods Ecol Evol 3: 792-796.

Wolak ME, Keller LF (2014). Dominance genetic variance and inbreeding in natural populations. In: Charmantier A, Garant D, Kruuk LEB (eds) Quantitative Genetics in the Wild. Oxford University Press: Oxford, UK, pp 104-127.

Wyman MJ, Stinchcombe JR, Rowe L (2013). A multivariate view of the evolution of sexual dimorphism. J Evol Biol 26: 2070-2080.

This work is licensed under a Creative Commons Attribution-NonCommercial-NoDerivs 4.0 Inter-
national License. The images or other third party material in this article are included in the article's Creative Commons license, unless indicated otherwise in the credit line; if the material is not included under the Creative Commons license, users will need to obtain permission from the license holder to reproduce the material. To view a copy of this license, visit http://creativecommons.org/licenses/bync-nd/4.0/

(C) The Author(s) 2017

Supplementary Information accompanies this paper on Heredity website (http://www.nature.com/hdy) 